\title{
Erratum
}

\section{Small Deformation of Normal Singularities}

\section{Shihoko Ishii}

Department of Mathematics, Sangyo-Ika University, Yahata-Nishi, Kitakyushu, 807 Japan

Math. Ann. 275, 139 (1986)

This note corrects a mistake in the proof of Lemma 3.1 in [1]. The isomorphism

$$
\operatorname{Ext}_{\mathscr{O}_{E_{*}^{\prime}}^{i}}^{i}\left(\mathcal{O}_{E_{*}^{\prime}}\left(-L_{r}+K_{E_{*}^{\prime}}\right), \mathcal{O}\left(K_{E_{*}^{*}}\right)\right) \simeq \operatorname{Hom}_{\mathscr{O}_{C^{*}}}\left(R^{n-i} f_{*} \mathcal{O}_{E_{*}^{*}}\left(-L_{r}+K_{E_{*}^{*}}\right), \mathscr{O}_{C^{*}}\right) \text {, }
$$

which was described at $l .9$ in p. 145 does not follow Theorem 11.2, $(f)$ (III, in [2]) immediately. Because we cannot apply the theorem to $\mathscr{O}_{C^{*}}$ which is not an injective module.

However the lemma remains true. In fact, in the original proof, the preceding isomorphism was used only to prove the assertions from $l .11$ to $l .13$ "there exists an integer $r_{0}$ such that for any $r \geqq r_{0}, f_{*} \mathcal{O}_{E_{*}^{*}}\left(L_{r}\right)=0$ and $R^{1} f_{*} \mathcal{O}_{E_{*}^{*}}\left(L_{r}\right)$ is zero (resp. torsion free) if $n \geqq 3$ (resp. $n=2$ )." So, we will show the above assertions by a correct method below.

Applying the relative duality theorem (III, 11.1 in [2]) to the flat morphism $E_{*}^{\prime} \rightarrow C^{*}$ of relative dimension $n-1$, we get

$$
\mathbb{R} f_{*} \mathcal{O}_{E^{*}}\left(L_{r}\right)[n-1]=\mathbb{R} \mathscr{H}_{\mathrm{om}_{\mathcal{O}_{C *}}}\left(\mathbb{R} f_{*} \mathcal{O}\left(-L_{r}+K_{E^{*}}\right), \mathcal{O}_{C^{*}}\right) \text {. }
$$

Note that there exists an integer $r_{0}$ such that, for any $r \geqq r_{0}, R^{i} f_{*} \mathcal{O}_{E_{*}^{*}}\left(-L_{r}+K_{E_{*}^{\prime}}\right)$ is 0 if $i>0$ and locally free if $i=0$ respectively. Since for $r \geqq r_{0}$, $\mathscr{E}^{\mathscr{2}} x t_{\mathrm{O}_{\mathrm{C}^{*}}}\left(f_{*} \mathcal{O}_{E_{*}^{*}}\left(-L_{r}+K_{E_{*}^{\prime}}\right), \mathcal{O}_{C^{*}}\right)$ is 0 if $j>0$ and locally free if $j=0$, which yields $R^{i} f_{*} \mathscr{O}_{E_{*}^{\prime}}\left(L_{r}\right)$ is 0 for $i<n-1$ and locally free for $i=n-1$ respectively. This completes the proof of our assertions.

\section{References}

1. Ishii, S.: Small deformation of normal singularities. Math. Ann. 275, 139-148 (1986)

2. Hartshorne, R.: Residues and duality. Lect. Notes Math. 20. Berlin, Heidelberg, New York: Springer 1966 